\title{
Design of Information Service System Based on RSS Technology Xiao Yu
}

School of Information Engineering, Wuhan Technology and Business University, Wuhan, Hubei Province, 430065

\begin{abstract}
This essay focuses on the development of RSS Information Technology combined with the features of information service system. From the general design of the system to functional analysis, and finally we design the framework. With the use of MVC design pattern during the design of the system, we made a design scheme on the basis of the RSS information service system.
\end{abstract}

Keywords: RSS; Information service system; System design; News subscription; MVC

\section{基于RSS技术的信息服务系统设计}

\author{
喻晓 \\ (武汉工商学院 信息工程学院, 湖北 武汉 430065)
}

摘要：本文针对信息技术 RSS 技术的发展，结合信息服务系统的特点。从系统总体设计到功能分析，最后进行架构设 计，在系统设计过程中采用了 MVC 设计模式，提出了一种基于 RSS 技术的信息服务系统设计方案。

关键词: RSS；信息服务系统；系统设计；新闻消息订阅；MVC

中图分类号： 文献标志码：A

\section{引言}

随着信息技术的到来，人们更多的从互联网上获取信息。RSS 技术就是信息时代的产物。它的特点包 括来源多样的个性化聚合特性; 信息发布的时效、低成本特性; 无垃圾信息，便利的本地内容管理特性。 因此, 通过利用 RSS 技术, 大家可以更方便的在网络上获取自己想了解信息。这样不仅缩短了用户在了解 新闻过程中花费的时间，同时能让用户可以在第一时间掌握最新消息。

\section{RSS简介}

RSS 自 1999 年由 Netscape 创建以来一直都是聚合技术的代表，焦玉英等著的网上合作数字参考咨询 的服务模式与管理机制提出一个较完整的基于 XML 流的 RSS 个性化内容聚合运行模型 [1-2]。自 2003 年以 来互联网上出现了一些中心化信息聚合服务商, 以 Feed 在线聚合、阅读为基础服务, 派生出多种服务方式 [3]。RSS (信息聚合)在电子商务网站中的应用方式:创建新型信息发布平台, 提供 RSS 推送服务; 提供个性 化 RSS 服务; 自动跟踪功能 [4]。

RSS 本身是一种 XML 格式[5]。RSS（Rich Site Summary）以建立开放、标准、简易的内容收集机 制及频道描述框架为目标, 一种可以共享新闻与其他 Web 内容的 XML 格式 [6]。一个 RSS 文件就是一段规 范的 XML（标准通用标记语言的子集）数据。RSS 订阅是一种方式, 即 Really Simple Syndication(简易 信息聚合)，它执行方式比较简单，主要被用于不同站点之间传递分享资料信息。基于 RSS 的内容聚合技 术解决了互联网中的站点之间内容共享的问题 [7]。RSS 技术广泛使用在网上新闻频道和 Blog, 通过使用 RSS 订阅功能大大加快了信息获取，在一个站点中增添 RSS 订阅功能，能使用户更快、更准确的获取到最 
新的新闻。普通用户可以通过电脑或者手机下载 RSS 阅读器, 能够随时阅读最新的新闻资讯。这种可以不 用打开网站详情页面就可以阅读网上新闻的方式受到众多好评。

\section{2 信息服务系统}

设计信息服务系统是基于 $\mathrm{B} / \mathrm{S}$ 结构的一个信息系统，集合了新闻订阅和文件上传下载功能，系统设计 在Windows7 操作系统平台下进行编程。通过设计该系统，用户不仅可以查看最新新闻资讯，还可以订阅 新闻资讯，并且方便的下载自己所需要的文件。

系统中使用 MVC 设计模式并基于 J2EE 平台的多层架构的设计。采用该方式可以使系统的扩展性较好。 带来的便利是在满足当前用户需求的同时还兼顾系统可能的功能扩充。本系统在设计的时候, 根据功能模 块互相独立的特性，将系统分成了不同的模块，并且在对这些模块进行管理的时候是集中式的。

系统的用户可以查看最新的新闻消息。当用户被新闻资讯吸引，并且想在最快时间内掌握最新新闻资 讯时系统可以提供的消息订阅功能。当用户对于相关文件有需求时, 用户可以浏览文件, 查看文件的详细 信息，可以对文件按时间或下载顺序进行查看，并且可以下载文件和发表对文件的评论。系统还可以增添 其他站点的 RSS 和对文件进行高效率的管理。

\section{3 系统设计}

\section{1 系统需求分析}

登录系统的角色包括系统管理员和普通用户。以下对每个角色需要实现的功能进行分析和说明。

1. 系统管理员

设计管理员登录功能, 主要是考虑到系统的安全性。信息服务系统中只有系统管理员才能发布新闻和 文件的上传和下载。系统管理员只有当验证通过后，管理员才能对系统进行管理。

由于本系统只有一个管理员，所以系统管理员有如下权利来管理系统：

(1) 新闻消息发布: 系统管理员可以随时发布最新新闻资讯

（2）文件的上传：文件上传功能仅对系统管理员有效，系统管理员在进入到文件管理页面中可以选择 添加文件，添加的文件包括对该文件的详细描述，文件的说明以及类型选择。

（3）文件管理：当管理员通过验证身份后，会获取对文件修改的权限，之后管理员可以添加文件，修 改文件以及删除文件。

(4) 添加 feed: 系统管理员可以通过添加其他新闻网站的 feed，将其发布的最新新闻资讯添加到自己 站点中，这样大大节省了发布相同新闻所浪费的时间。

(5)生成自己的 feed: 当系统管理员在添加了许多网站的 RSS 后, 为了方便用户在本网站中方便读取 其他站点的新闻资讯，于是与我们就将添加的 RSS 统一起来，然后生成自己 RSS，这样一来，就实现了不 同网站之间的新闻的统一。

2. 普通用户

(1) 新闻浏览:用户可以根据自己喜好浏览新闻。

(2) 订阅喜欢的新闻资讯：当用户看到一个自己感兴趣的新闻资讯，并且想实时了解新闻事件的发展 情况, 这里我们提供了一个新闻订阅功能, 用户只要通过这个功能, 就能够随时获取到最新的新闻动态。

(3) 文件查看: 进入网站首页, 页面中会罗列出数据库中已经存在的文件, 用户可以清晰明白的看到 
文件名、文件大小和更新日期、以及文件的下载次数和系统管理员对该文件的详细描述。用户可以根据自 己需要, 查看或下载文件。

（4）文件搜索：文件搜索功能在任何系统中都存在，它是一个简单的功能，具体是当用户进行文件搜 索的时候, 根据用户输入的要查询的内容的关键字, 系统会与数据库中的数据进行比对, 同时将搜索到的 并带有和关键字相同的文字的文件的结果显示出来。用户通过此功能，来找到自己所需要的文件。

(5) 文件下载：本系统还提供了文件下载功能，当用户找到需要下载的文件时，只需点击下载，系统 就会弹出要下载的文件的对话框, 用户可以根据自己需要, 有选择性的选择下载路径, 文件在下载完成后, 文件的下载次数就会加 1 。

\section{2 系统总体设计}

信息服务系统设计是基于新闻消息订阅功能的，并且通过 RSS 技术来实现。在设计过程中，增添了文 件的上传和下载的功能。RSS 技术实现了多源信息的个性化聚合。[8] 网络原生数字资源管理者在生成 RSS 文档之后, 会对其进行主题聚合, 然后再加入到 RSS 提要库中。此后, 就需要内容集结器发挥其 作用了 $[9]$ 。

普通用户可以查看新闻资讯，同时订阅喜欢的新闻，还可以查看文件，对文件进行排序，并且用户还 可以对文件进行评论, 系统管理员与普通用户相比, 可以发布新闻, 将其他站点的 RSS 添加到本站点中, 同时生成自己站点的 RSS, 也能对文件进行有效删除, 适当修改, 不重复添加等操作。普通用户可以直接 进入文件下载管理系统的首页，进入首页后就可以对文件进行排序。一方面可以通过下载次数的多少排序， 另外一方面可以根据最新系统管理员的推荐进行排序。文件搜索同时还可以对文件评论。系统管理员进入 到管理首页需要验证登录, 只有登录成功后才能对系统进行管理。系统中管理员管理的功能具体包括新闻 消息的发布，解析其他站点的 RSS 源，已经发布的文件修改，新的文件的添加以及已经存在的文件删除。

通过对上述系统的分析, 可以画出系统的功能模块图, 如图 1 所示。

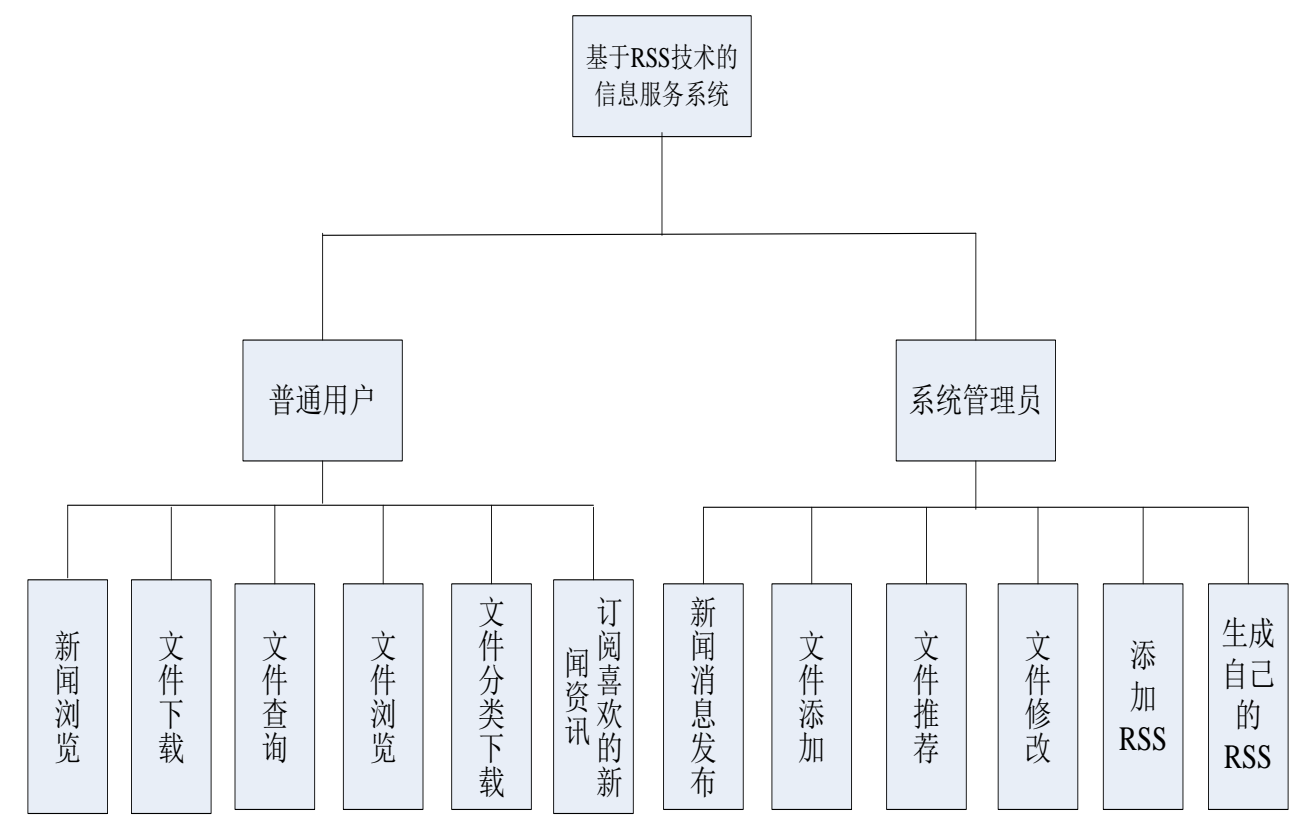

图 1 系统功能模块图 
通过系统设计, 用户可以订阅和查看最新新闻资讯, 并且可以方便的下载自己所需要的文件。在网页 设计代码中添加 RSS 的语句。 RSS feed, 亦可称为 RSS 种子或 RSS 文件, 其形式就是一段标准的 XML 格 式数据, 它通常括〈channe1〉,〈image〉, 〈item〉,〈textinput〉等 4 个元素 [10]。

\section{4 结语}

在本系统中用到了 RSS 技术，通过 RSS 技术很容易的实现了信息聚合。本系统设计的是基于 $\mathrm{B} / \mathrm{S}$ 结构 的一个信息服务系统，它集合了新闻订阅和文件上传下载功能。

\section{参考文献:}

[1］程＼cjkstart莉，刘东苏．基于 RSS 的个性化信息服务模型研究 [J]．现代情报，2013，33（2)：47-51.

[2] 焦玉英，项英．网上合作数字参考咨询的服务模式与管理机制 $[\mathrm{J}]$. 图书情报知识，2005，(8)：43-45.

[3］全国图书馆参考咨询联盟[EB/OL]. http://www. ucdrs. net/admin/union/index. do, 2012- 05- 28.

[4] 尹倬. 合作式数字参考咨询系统的比较研究一以 “网上联合知识导航站” 和 “联合参考咨询网” 为例 $[J]$. 图书馆 工作与研究，2009, (8)：70-73.

[5] 陈 宁, 徐志明. Web3.0 时代基于 RSS 的网络原生数字资源推送流程分析 [J]. 农业图书情报学刊, 2014, 26(12)： $26-28$.

[6] 陈建. 基于 RSS 技术的移动图书馆阅读推荐系统设计研究 [J]. 山东工业技术，2014，238(20)：140.

[7] 宋欣辉. 基于 RSS 元数据的地方志资源推送 [J]. 电子科技技术. 2016, 3(04):524-526.

[8］陈 荣．基于 RSS 技术的社区信息服务系统设计 [J]．赤峰学院学报（自然科学版），2016，32(7)：22-23.

[9］黄艳娟. 基于 RSS 的图书馆个性化信息推送服务 [J]。情报科学, 2006, (7) :1023-1026.

[10］吴振兴. RSS 元数据在门户网站建设中的应用 [J]. 现代图书情报技术, 2004, (10): 60-64.

\section{References:}

[1] Cheng Li, Liu Dongsu. Study on Personalized Information Service Model Based on RSS [J].Modern Information, 2013, 33 (2):47-51.

[2] Jiao Yuying, Xiang Ying. Service Mode and Management System of Online Collaborative Digital Reference [J].Document, Information and Knowledge, 2005, (8):43-45.

[3] National Coalition of Library Reference [EB/OL]. http://www.ucdrs.net/admin/union/index.do, 2012-05-28.

[4] Yin Zhuo. Comparative Study on Collaborative Digital Reference Service System-a "Joint Knowledge Online Navigation Station" and "Combined Reference and Consultation Network" as An Example [J].Library Work and Study, 2009, (8):70-73.

[5] Chen Ning, Xu Zhiming. Push Process Analysis of Network Raw Digital Resources Based on RSS in Age of Web3.0 [J].Journal of Library and Information Sciences in Agriculture,2014,26(12):26-28.

[6] Chen Jian. Research on Recommendation System Design of Mobile Library Reading Based on RSS Technology [J].Shandong Industrial Technology,2014,238(20):140.

[7] Song Xinhui. Local History Resources Push based on RSS meta data [J].Electronic Science and Technology, 2016, 3(04):524-526.

[8] Chen Rong. System design of Community Information Service Based on RSS Technology [J].Journal of Chifeng College (Natural Science Edition), 2016, 32(7):22-23.

[9] Huang Yanjuan. Library's Individualized Information Push Service Based on RSS [J].Information Science, 2006, (7):1023-1026.

[10] Wu Zhenxing. RSS Metadata in Application of Portal Site Construction [J].New Technology of Library and Information Service, 2004, (10):60-64. 